The paper trail to commercialization

\author{
Jim Kling \& Laura DeFrancesco \\ A survey of Nature Biotechnology's authors reveals many paths to commercial success.
}

$\mathrm{N}$ ature Biotechnology claims to be the journal "that brings research out of the laboratory and into the marketplace." But how many of the papers published in our pages ultimately see the light of day in commercial enterprises? And what are the paths that research takes on its way to becoming successful products?

Last year, as part of our $10^{\text {th }}$ anniversary 을 celebration, we contacted authors who had published research with us over the previous ten years to evaluate how (and whether) their work had been commercialized. Judging by the number of respondents, the majority of research that we publish never is directly carried forward into products. This is not surprising-making it in the marketplace is just one measure of a successful research program; some work, no matter that it is groundbreaking and conceptually interesting, simply won't translate into a product or drug (or only translates many years later after another advance removes a hurdle to commercialization). Conversely, work described in papers that receive relatively few citations can sometimes turn into commercial gold.

We present here the experiences of several authors (who responded to our survey) attempting to commercialize their work. We follow their stories - what has happened to the discoveries in the years since they published them and what paths they took to put their ideas and inventions into the public domain. We made no attempt to capture all such programs from our large list of authors. Thus, this is not meant to be a comprehensive look at what has transpired from all the work we publish, just a snapshot.

More than half of those we spoke with have been involved in starting a company and a number either are joining, or will soon join, the ranks of serial entrepreneurs. Several discoveries

Jim Kling is a freelance writer based in Bellingham, Washington. Laura DeFrancesco is Senior Editor at Nature Biotechnology. have been packaged up as research tools, and can be purchased today. Other researchers have translated their original findings into human therapeutics that are now wending their way through clinical trials.

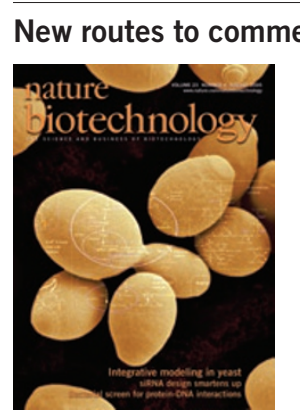

preclinical toxicology testing. Raising earlystage venture capital is incredibly difficult, according to Hancock. "Venture capitalists are no longer sold on the romance [of biotech]. Early stage [seed investment] is not worth having because it's equivalent to giving away a company and idea for $\$ 1$ million," he says.

Nature Biotechnology has been the conduit for two Hancock technologies that are being taken forward. In a 2005 paper, his laboratory reported on a system for highthroughput screening of antimicrobial peptides $^{1}$. Peptides with antimicrobial activity, although ubiquitous in nature, are costly to produce and thus manipulate and optimize. But in 2005, Hancock's group reported on a high-throughput luciferase monitoring system that allows them to make and test peptides by the thousands. Hancock's latest patent application describes over 100,000 novel peptides. In the 2005 paper, the group started with a 12 amino acid long-peptide, and systematically replaced each amino acid with the other 19 , created double and triple replacements and measured the activity of each. This, Hancock says, led to the isolation of the smallest peptide with broad-spectrum anti-microbial activity, which is now being developed by the program that is supported by the Gates Foundation.

More recently, Hancock described in our pages the isolation of a peptide with the ability to harness innate immunity by upregu-lating the production of chemokines ${ }^{2}$, which in turn recruit monocytes and macrophages to the site of

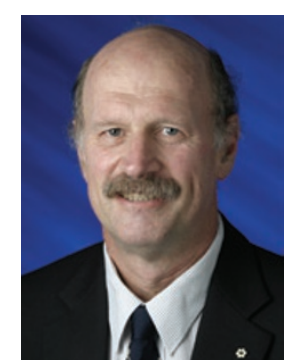

Serial entrepreneur Bob Hancock sticks with basic biology. Without a basic understanding of mechanism, a drug will never make it through development. at UBC, designed to take products through 
an infection, particularly useful in people with compromised immune systems. The beauty of this peptide is that its mechanism of immunomodulation appears to be entirely different from that of previously described peptides, which can induce inflammation, counteracting any potential benefit. This finding, too, has led to patent applications, one of which has been licensed exclusively to Inimex, whereas others are being developed through the Grand Challenges program. Hancock has great hopes for these peptides; the Inimex-licensed peptides are almost ready to go into the clinic.

According to Barbara Campbell, who, as associate director of the University Industry Liaison Office at UBC, has been handling Hancock's portfolio for the past seven years, establishing commercialization strategies has been a collaboration between her office and Hancock. Once an inventor gets involved with a company, the tech transfer office gets interested, but once multiple companies are involved, then they really have to step up and make sure that there are no areas of overlap that can be costly in time and legal fees. Although working with Hancock has created a lot of work for her, he makes her job easier, as his extensive experience makes him sensitive to these potential pitfalls.

Despite all this commercial activity, Hancock describes himself as a basic researcher. He says he's learned from experience that without a basic understanding of mechanism, a potential drug will never make it through development. And when Bob Hancock speaks, people listen-he has sat on no fewer than 17 scientific advisory boards, a clear sign that his expertise is appreciated by his peers.

1. Hilpert, K. et al. High-throughput generation of small antibacterial peptides with improved activity. Nat. Biotechnol. 23, 1008-1012 (2005). (This paper has been cited 27 times according to Thomson ISI Web of Knowledge. In the references following, the number of citations appearing in parentheses at the end are all based on Thomson, October 12, 2007.)

2. Scott, M.G. et al. An anti-infective peptide that selectively modulates the innate immunity response. Nat. Biotechnol. 25, 465-472 (2007). (3 citations)

\section{Out of left field, into the mainstream}

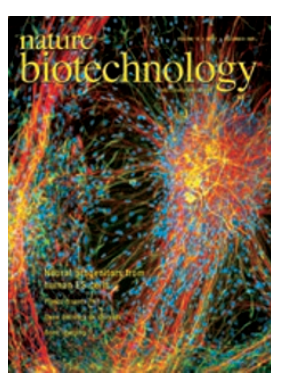

Group II introns are not the stuff of headlines, but for the past 15 years, University of Texas's (UT; Austin) Alan Lambowitz has been working away at these tiny mobile elements. His work has spawned an actual product-TargeTron, a gene disruption kit sold by Sigma Aldrichnumerous patents, a company and a raft of

publications showing how introns can be used to engineer otherwise recalcitrant, yet clinically important species like the human pathogens Staphylococcus aureas ${ }^{1}$ and Clostridium difficile ${ }^{2}$.

Having found that group II introns_-which are self-catalytic mobile genetic elementsintegrated into DNA through base pairing, Lambowitz demonstrated in his 2001 Nature

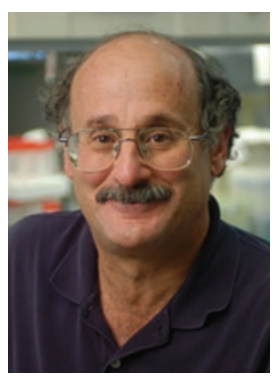

Alan Lambowitz is gearing up to expand his gene targeting technology into new areas. combination with an associated intron-encoded DNA endonuclease, introduces double-stranded breaks in the DNA, creating a template for homologous recombination, similar to the mechanism of zinc-finger endonucleases.

Since then, Lambowitz's group has improved the efficiency of integration tenfold, using a computer algorithm to optimize integration, which purchasers of TargeTron can use to design their own introns. And they (and others) have racked up an impressive number of species as well as genes that have been targeted with this system-some 45 genes and 16 different bacterial species are listed on the TargeTron pages on the Sigma Aldrich (St. Louis) website (http://www. sigmaaldrich.com/Area_of_Interest/Life_ Science/Functional_Genomics_and_RNAi/ TargeTron.html).

Lambowitz acknowledges that the system hasn't reached its stride yet. "It comes from out of left field," he says. But he still thinks that its time will come-after all, although the technology is now six years old, the kit has been available only for two years, work on expanding the host range has come out just this year, and more importantly, he feels its advantages have yet to be fully appreciated. The most important among them, according to Lambowitz, is that group II introns can be used in species without well-developed genetic systems, as demonstrated independently by the work of Nigel Minton, at the University of Nottingham, $\mathrm{UK}^{2}$. Minton has developed a set of introns that he has used to target several Clostridium species, starting with the TargeTron kit (he calls his introns "the Clostron"2), and testifies on his website to the importance of the gene disruption technology. "Twenty-one genes in a few short months equivalent to [the] last 20 years [of] published achievements" he writes. (http://hcai.nottingham. ac.uk/minton.pdf)

Lambowitz's laboratory has recently scored a major breakthrough for the technology by showing that group II introns can target mammalian genes. And given their ability to generate double-stranded breaks, this provides a potential alternative to zinc-finger nucleases for initiating homologous recombination and gene replacement, with a technology that might be more accessible than zinc fingers. "[People] don't need trade secret zinc-finger libraries. Anyone who goes to Sigma Aldrich or our website can design an intron that can insert," says Lambowitz.

The path to commercialization started at Ohio State University (Columbus), where some of the early work was done, resulting in the creation of a licensing entity, Ingex (St. Louis), which is still in business today and holds technology described in the Nature Biotechnology paper in its intellectual-property portfolio. Moving to the University of Texas in Austin, where Lambowitz now heads the university's Institute for Cellular and Molecular Biology, did create some interesting challenges, but Lambowitz declines to elaborate because the three entities (the two universities and Ingex) have since worked out an arrangement that suits them all.

And UT is now looking at a second generation of startups, based on the expanding Ingex portfolio, which, according to Greg Pogue, UT's technology transfer manager for the Lambowitz work, will take the technology to the next level and into applications as diverse as cancer and biofuels. According to Pogue, the technology has stepped over a significant barrier, in demonstrating that it can be applied to both bacterial and eukaryotic genome engineering. This opens up a host of possibilities and Pogue, himself a former biotech researcher and executive with 35 publications and 12 patents to his name, is using his contacts in the industry to identify appropriate partners for developing particular applications.

1. Yao, J. et al. Use of targetrons to disrupt essentia and nonessential genes in Staphylococcus aureus reveals temperature sensitivity of LI.LtrB group II intron splicing. RNA 12, 1271-1281 (2007).

2. Heap, J.T. et al. The ClosTron: A universal gene knockout system for the genus Clostridium. J. Microbiol. Methods 70, 452-464 (2007).

3. Karberg, M. et al. Group II introns as controllable gene targeting vectors for genetic manipulation of bacteria. Nat. Biotechnol. 19, 1162-1167 (2001). (31 citations) 


\section{Industrial R\&D glimpsed}

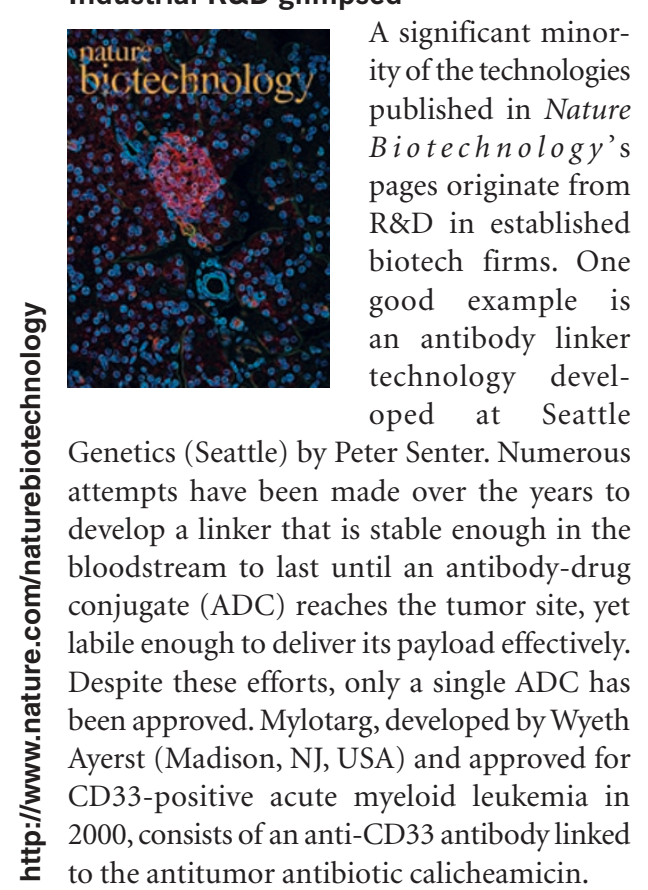

The conjugate works in part because it attacks a blood-borne malignancy and the antibody can find its target quickly, before its systemic drug release leads to decomposition and toxicity. "It can saturate the tumor within 30 minutes, so the requirement for stability is not nearly as high as for tumors that are less accessible," says Senter, who is vice president of chemistry at the company.

In his team's 2003 Nature Biotechnology paper ${ }^{1}$, Senter and his colleagues describe a novel peptide linker designed to be cleaved by the enzyme cathepsin B, which is present within cell lysosomes. Most traditional linkers, includ-

ing Mylotarg, are based on acid-labile linkers that are designed to release drug within acidic vesicles or in the acidic environment of tumors. However, they are somewhat unstable even outside of tumors, causing release of the active drug and hurting the conjugate's specificity.

Seattle Genetic's ACD proved to be more specific and less toxic than previously reported ADCs, with a half-life in animals of 7-10 days, compared with typical half-lives of $1-3$ days. Senter believes his company's approach addressed previous shortcomings. "A lot of the linkers used were too unstable. Others had flaws with the antibodies that were used. And a lot of the drugs used in the conjugates weren't potent enough to kill tumor cells effectively. We addressed each one of those problems in the 2003 Nature Biotechnology paper," Senter says.

The approach was taken forward by Seattle Genetics to develop a novel drug to pair with its linkers. It licensed a pentapeptide called auristatin E from Arizona State University natu-

ral product chemist George Pettit. Auristatins inhibit microtubial polymerization similar to how vinca alkaloids do, but more potently, Senter says. His team then developed synthetic derivatives of auristatin to prepare them for conjugation, and to tweak other properties.

"To go to a more stable linker required an understanding of the enzymes that are involved in drug activation, and precisely where they exist," says Senter. Biochemical studies showed that the primary enzyme responsible for cleaving peptide linkers is cathepsin $\mathrm{B}$, which has minimal activity in the bloodstream. "That's why it's so much more stable than previous linkers." The combination of a stable linker and highly potent drug makes the conjugate both safe and effective, according to Senter.

The team compared the peptide linkerbased conjugates to acid-labile conjugates. The peptide conjugate led to regressions and cures of tumors in mice at well-tolerated doses.

Although larger firms tend to regard promising technology as trade secrets, publishing Senter's work rather than keeping it under wraps was fully compatible with Seattle Genetics' strategy of raising company visibility, according to Peggy Pinkston, associate director of communications. To Pinkston the benefits are tangible.

"Otherwise," she says, "companies [such as Seattle Genetics] couldn't attract ambitious and talented scientists."

The linker is a key component of one of Seattle Genetics' lead compounds, SGN-35, which is in phase 1 clinical trials for the treatment of Hodgkin's disease and other $\mathrm{CD} 30^{+}$ malignancies. The company is also

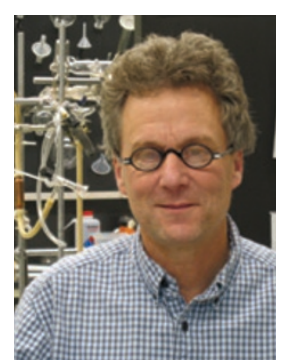

Seattle Genetic's Peter Senter addressed shortcomings of antibody linkers, leading to a drug in clinical trials as well as revenue for his company.
1. Doronina, S.O. et al. Development of potent monoclonal antibody-auristatin conjugates for cancer therapy. Nat. Biotechnol. 21, 778-784 (2003). (57 citations)

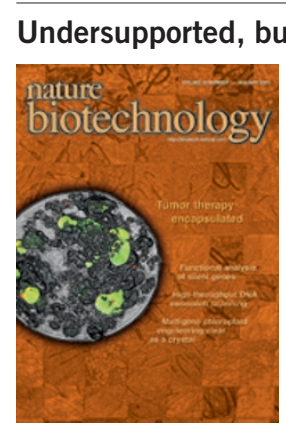

\section{Undersupported, but undaunted}

Henry Daniell is not easily discouraged. After all, his invention-a universal chloroplast vector, which he first reported on in our pages in 1998 (ref. 1) - is now patented in 15 countries, thanks in no small measure, he thinks, to the validation it received by surviving the review process. What's not so good for Daniell and everyone else trying to commercialize a product from transgenic plants is the enormous cost of bringing such a product to market, especially considering the difficulty of obtaining the necessary capital.

But on the surface, things are going well. Recent work from his laboratory and elsewhere has demonstrated that human proteins like insulin and interferon can be made in plants with engineered chloroplasts at levels not possible with nuclear trangenes. In a later Nature Biotechnology paper $^{2}$, he reported that $45 \%$ of tobacco leaf protein was derived from a transgene, the insecticidal protein cry2Aa2, representing the highest level of foreign gene expression ever reported in plants at the time. Since then, equally impressive levels of insulin and interferon have been achieved using the system $^{3,4}$. Low expression levels is the thing that Daniell feels has held back the field of plantmade pharmaceuticals_ or at least one of the things. And with his chloroplast vector, which inserts into all 10,000 copies of the chloroplast genome, he feels he has that problem solved.

Another virtue of targeting the chloroplast genome with a transgene is that containment is not an issue. Because the chloroplast genome is maternally inherited, pollen won't disseminate the transgene. And with tobacco, one of the Daniell systems, at the time of harvest, there are no flowers (they harvest leaves), so no reproductive organs are involved, which should make regulators breathe a sigh of relief. In fact, four transgenic plants have been approved for field trials, and the results of one such trial (insulin) have recently been reported ${ }^{5}$.

Daniell thinks he can further improve the odds of success with plants by delivering the expressed protein in plant powder. He recently showed that oral delivery of powdered plant cells expressing human proinsulin protected non-obese diabetic mice against development of insulitis. This could be a boon to 


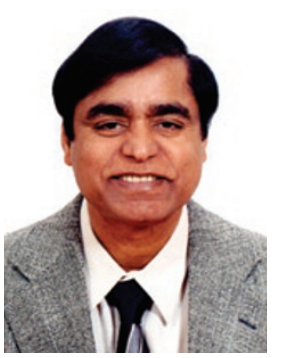

Henry Daniell says that having a cover photo in two highimpact journals raised the visibility of chloroplast engineering technology.

million in phase 1 to advance four chloroplastderived therapeutics from the Daniell laboratory that have been shown to be functional in animal studies. In keeping with Daniell's personal interest in solving problems in the developing world-he was born in India-GenCrest is working on chloroplast-derived vaccines.

All this sounds good. But with only $\$ 20$ million in the bank, Daniell knows it's not going to be enough to take any biopharmaceutical product through clinical trials. So even with the imprimatur of venture capitalists, he's still a long way from where he needs to be. But Daniell goes on undeterred-moving antigens for cholera, malaria, amebiasis, rotavirus and tuberculosis into plants, and hoping for the day that these will all make their way to the people who most need them.

1. Daniell, H. et al. Containment of herbicide resistance through genetic engineering of the chloroplast genome. Nat Biotechnol. 16, 345-348 (1998). (154 citations)

2. De Cosa, B. et al. Overexpression of the Bt cry2Aa2 operon in chloroplasts leads to formation of insecticidal crystals. Nat. Biotechnol. 19, 71-74 (2001). (125 citations)

3. Ruhlman, T. et al. Expression of cholera-toxin B-proinsulin fusion protein in lettuce and tobacco chloroplasts - oral administration protects against development of insulitis in non-obese diabetic mice. Plant Biotechnol. J. 5, 495-510 (2007).

4. Arlen, P.A. et al. Field production and functional evaluation of chloroplast-derived interferon- $\alpha 2 \mathrm{~b}$. Plant Biotechnol. J. 5, 511-525 (2007).

5. Daniell, H. Transgene containment by maternal inheritance: effective or elusive? Proc. Natl. Acad. Sci. USA 104, 6879-6880 (2007).

\section{Timing is everything}

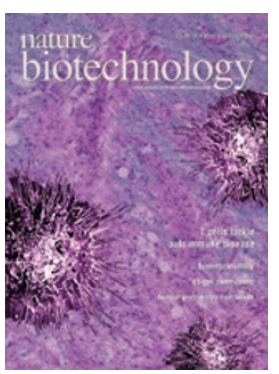

In 2002, Ryzard Kole described in our pages a mouse assay for testing antisense oligonucleotides ${ }^{1}$. The assay provides incontrovertible evidence, Kole says, that a particular oligonucleotide works by an antisense mechanism, something that has not always been so clear with antisense. In addition, it allows investigators to track the location and function of oligos injected into whole animals. In his Nature Biotechnology paper, Kole and his team designed an oligonucleotide to target a splice variant of green fluorescent protein (GFP). When this oligonucleotide was injected intraperitoneally into mice transgenic for the GFP splice variant, restoration of proper protein splicing and function could be confirmed by the detection of fluorescence in mice tissues, such as colon, small intestine and liver. This elegant assay provided the starting point for Kole to move therapeutic programs forward. But it hasn't been an easy path.

For one thing, although the biology might have been ready back then, the chemistry of the oligos was not. In the intervening years, nucleic acid chemistry has advanced to the point that one can make molecules that are stable in vivo. But Kole didn't have that at the time. And now that he does, the world both of researchers and the money men who back them have moved onto sexier therapeutic nucleic acid formats of higher potency, like RNA interference and microRNA.

But Kole remains undeterred, believing that targeting splicing is "a new frontier" and the company he founded back in 2001, Ercole Biotech (Research Triangle Park, NC, USA), is holding its own, according to Scott Forrest, the technology manager at the University of North Carolina, where all this has been playing out. It has space, a staff of three researchers, and a set of collaborations with companies, which is giving it a foothold in the commercial sector. From Forrest's vantage point, the company is independent, sound and secure. "It's a pleasure to see," he says. And without a dime of venture capital, which can be both good and bad, says Forrest. The university's shares in Ercole have not been diluted, but the company doesn't get what venture capitalists bring to the table, in terms of expertise in forming and managing a startup.

Getting there required the university taking the risk in providing early venture money, which has paid off in Ercole's case. "We don't always get it back, but if every once in awhile it leads to a living, breathing company, that's consistent with our larger mission of economic development, an attitude that has helped Research Triangle Park and the region make headway in becoming a biotech hotspot," says Forrest.

Ercole's raison d'être is to apply antisense technology to modulating alternative splicing and correcting splicing defects. Some $70 \%$ of genes undergo alternative splicing, and a number of serious diseases are caused by errors in splicing. Kole wants to target these diseases, and he's using the assay described back in 2002 to try to get there. To choose what diseases to target, he lets the chemistry be his guide. $\mathrm{He}$ says "[you can] see where oligos with a certain chemistry go — which tissues, which cells — and because of that, can chose what is a target gene and a target disease that we know we can treat because that's where the oligo goes."

Earlier this year, Ercole announced a collaboration with the antisense company AVI Biopharma (Portland, OR, USA) for developing therapies for Duchenne's muscular dystrophy and beta-thalassemia. Ercole also has a longstanding deal with Isis (Carlsbad, CA, USA) and a recent agreement with Santarus (San Diego), which affords them access to various oligonucleotide chemistries. Kole says he gets frequent requests from companies and indi-

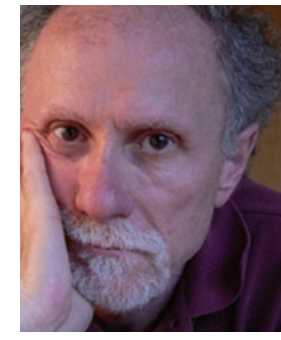

Ryzard Kole gets many requests for his oligo tracking technology, which he is using to develop therapies for diseases caused by aberrant splicing. vidual investigators to test their oligos and delivery systems.

The idea of using antisense to correct splice variants may soon be validated in humans. After the demonstration that systemically delivered oligonucleotides that target splicing have therapeutic effects in a mouse model of Duchenne's muscular dystrophy ${ }^{2}$, a Dutch biotech company, Prosensa (Leiden), received permission from the Netherlands Central Committee on Research to initiate a trial in humans using locally administered antisense oligos.

1. Sazani, P. et al. Systemically delivered antisense oligo mers upregulate gene expression in mouse tissues. Nat. Biotechnol. 20, 1228-1233 (2002). (94 citations)

2. Lu, Q. L. et al. Functional amounts of dystrophin produced by skipping the mutated exon in the $m d x$ dystrophic mouse. Nat. Med. 9, 1009-1014 (2003).

\section{Too far ahead of the curve?}

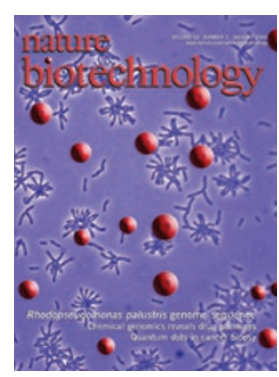

Some might say John Frangioni was ahead of his time when he published his 2001 article on diagnostic imaging agents to pinpoint osteoblast (bone-forming) activity $^{1}$. Six years have passed, and it's still "the cart before the horse," as he puts it.

The paper describes near-infrared (NIR) fluorescing derivatives of bisphosphonates, 
which are used clinically to inhibit demineralization by osteoclasts and shift the balance between mineralization and demineralization to bone formation. These labeled bisphosphonates bind to hydroxyapatite (HA), which is deposited by osteoblasts during bone formation, therefore revealing osteoblastic activity. Frangioni and his team chose the NIR part of the spectrum because biological molecules have minimum absorbance spectra and scatter in this region. Frangioni's team demonstrated the technology by studying the growing skeletal structure in living mice.

They went on to demonstrate a variety of nonskeletal applications of the agents. One NIR fluorophore derivative was shown

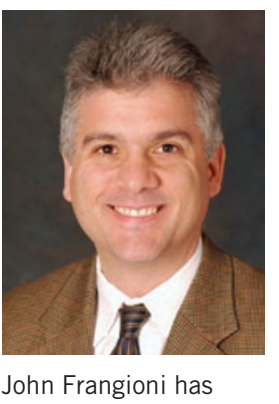

waded into the fray, building his own intraoperative imager to guide surgeons in real time.

sition in coronary calcification, which is an early stage of atherosclerotic lesions.

In 2002, the Beth Israel Deaconess Medical Center in Boston licensed the agents to VisEn Medical (Woburn, MA, USA), which has an in vivo fluorescence tomographic imaging system that it sells as an independent product. The company also performs contract research. Frangioni's agents are one of a suite of reagents that can be used with the system, which collects noninvasive images of osteoblast activity in research animals. Pharmaceutical and biotech companies developing bone-altering agents (for osteoporosis or bone healing, for example) find it useful in analyzing animal models.

But Frangioni has a grander vision. HA deposition isn't just involved in bone deposition. In addition to the aforementioned applications, Frangioni's agent can detect HA in microcalcifications found in breast cancer tissue and help distinguish benign microcalcifications, which contain predominantly calcium oxalate, from malignant ones, which contain more HA. The trouble is that his technology is way out in front of potential applications - there isn't a medical imager on the market that can make use of his reagents in the clinic. Frangioni would like to see his agents developed, but investors have turned a deaf ear. "Optical imaging hasn't been well vetted yet. It's a risk. Five years from now, it will be moot, because there will be several clinical optical imaging systems out there. At that point, no one will flinch [over funding development of imaging agents]", he says. Developing those agents is no small endeavor. They require current good manufacturing production and US Food and Drug Administration (FDA) approval and manufacturing certification. And with no reliable market, there has been no movement. "Imaging agents are stuck," he says.

So rather than waiting patiently for others to develop an imager, Frangioni has waded into the fray himself. His team has built an intraoperative NIR fluorescence imager designed to guide surgeons in real time. With the appropriate NIR fluorescent contrast agents, such imaging systems, agents could improve sentinel lymph node mapping, tumor excision and help surgeons avoid vessels, nerves and other critical structures. The device, which was the subject of a 2004 Nature Biotechnology article ${ }^{2}$, consists of a box with a swinging arm that can be positioned like a lamp. The monitor shows a color image of the patient, overlaid by two NIR channels, which can be used with imaging agents to highlight specific structures. For example, one channel could show tumor tissue marked with one agent, whereas the other might light up nerve cells highlighted by another agent.

In 2003, Beth Israel issued a nonexclusive license to GE Healthcare (Waukesha, WI, USA) for clinical development of the intraoperative imaging system. In the meantime, Frangioni's group is pressing forward with its own development. His laboratory has constructed its own state-of-the-art operating room in which to test the imaging system, with engineers, surgeons and chemists working together to improve the technique. As Nature Biotechnology goes to press, he expects to have begun two phase 1 clinical trials, one for sentinel lymph node mapping in lung cancer patients and another one for use with reconstructive plastic surgery.

Frangioni's overarching goal is to develop the imager as a platform technology for application-specific NIR fluorescent contrast agents. "Until the imaging systems are there, the agents don't have a use," he says.

1. Zaheer, A. et al. In vivo near-infrared fluorescence imaging of osteoblastic activity Nat. Biotechnol. 19 1148-1154 (2001). (66 citations)

2. Kim, S. et al. Near-infrared fluorescent type II quantum dots for sentinel lymph node mapping Nat. Biotechnol. 22, 93-97 (2004). (245 citations)

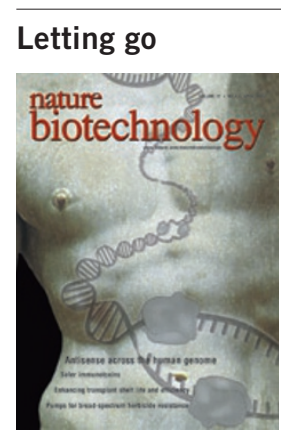

If there's anything that Dallas' University of Texas Southwestern's Ellen Vitetta has learned in the years since her 2003 Nature Biotechnology paper $^{1}$ was published, it is when and how to let go. In that paper, Vitetta solved a problem that had been plaguing those developing immunotoxins and cytokines as human therapeutics-vascular leakage syndrome. Ricin-based immunotoxins, in particular, were being tested in patients with chemorefractory lymphoma, and although the patient response had been encouraging, the side effects, principally vascular leakage, limited the dose that could be used. In her paper, Vitetta and her team genetically engineered the ricin A chain in a region that she suspected caused vascular leakage and isolated one variant that retained its toxicity, yet eliminated its tendency to induce vascular leakage ${ }^{1}$. What that meant practically is that the dose of the toxin used in patients could be increased, improving the therapeutic index, "giving more bang for the buck," as Vitetta describes it.

But as anyone working on human therapeutics knows, the steps that follow a drug's development can be difficult, expensive and at times frustrating. Vitetta thought she was getting close to the clinic when she obtained a National Institutes of Health grant to ramp up for phase 1 clinical trials and had found a collaborator at the National Cancer Institute to do the trial itself essentially for free. But it wasn't to be, as the FDA, citing safety reasons, required more testing than could be supported by the funds she had available. And in the course of trying to work out a way forward, Vitetta's group found that the mutated ricin A chain was less stable than the unmodified version.

That was a setback for the project, but Vitetta eventually found a commercial sponsor to take on the stability problem and carry on with clinical trials, and now she feels the program has a reasonable chance of success. But that also means that she has lost control. "It will go where it goes when they decide it will go. The good news is that at least it didn't die on the vine. I'm confident that once the process development has been completed it will perform very well," she says.

In the meantime, other biologic cancer therapies, such as Brentford, UK-based GlaxoSmithKline's Bexxar (tositumomab) 
Genentech's (S. San Francisco, CA, USA)/ Biogen-Idec's (Cambridge, MA, USA) Rituxan (rituximab) and Biogen-Idec's Zevalin (ibritumomab) have been approved, which might make it tough to recruit patients for clinical trials. Yet, Vitetta says although these are all great drugs, people relapse, so there is a need for therapies to treat them. "You can't count on one antibody to hold a tumor at bay forever. It just isn't going to happen," she says. And in fact, she has completed a phase 1 trial with a product that is a mixture of immunotoxins, with promising results. Vitetta waxes philosophical when it comes to her role in getting these drugs into the clinic. "I've learned in this business that there's only so much you can do before you have to give up control. Before I let it go, at least we show the company it is a good drug candidate that actually works in humans," she says.

But that's not the end of the story. Vitetta moved her mutated ricin along a parallel

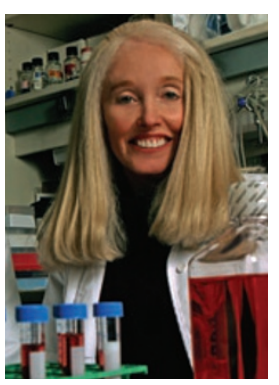
trajectory, which has had greater success-a ricin vaccine, which has captured some biodefense funding and is being moved forward as well. By introducing a second mutation that knocks out toxicity of the ricin Ellen Vitetta found that the path to a successful drug can be difficult, expensive and frustrating, but overall worthwhile.

that passively protected mice that were challenged with ricin ${ }^{2}$. They have furthered this project by developing a model for aerosolized and ingested ricin $^{3}$, the more likely route from a bioterror attack, and have obtained funds to test the vaccine with adjuvant in a second clinical trial. "If the money holds up, we'll move toward advanced trials with our licensee," she adds.

Summing up the years since her 2003 publication, Vitetta comments, "It brought licensing fees from several companies, we moved forward with several grants and learned a heck of a lot about vaccinology and recombinant protein production. Looking back, it was a worthwhile journey and hopefully we will have developed two products that will eventually be approved by the FDA."

1. Smallshaw, J.E. et al. Genetic engineering of an immunotoxin to eliminate pulmonary vascular leak in mice. Nat. Biotechnol. 21, 387-391 (2003). (36 citations)
2. Vitetta, E.S. et al. A pilot clinical trial of a recombinant ricin vaccine in humans. Proc. Nat. Acad. Sci. USA 103, 2268-2273 (2006).

3. Smallshaw, J.E. Richardson, J.A. \& Vitetta, E.S. RiVax, a recombinant ricin subunit vaccine protects mice against ricin delivered by gavage or aerosol. Vaccine 25, 7459-7469 (2007)

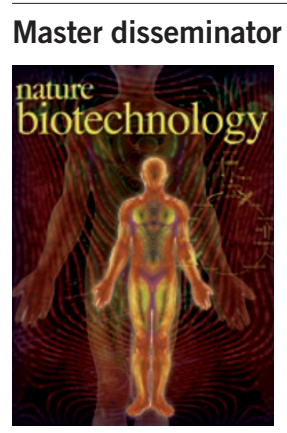

Mas s a chusetts Institute of Te c h n o logy's (Cambridge, MA, USA) Dane Wittrup is one of the more prolific of those responding to our survey, with five publications in Nature Biotechnology in the past ten years. But he harkens back to one of his earliest as having the largest impact on his field of protein engineering. In 1997, he and a graduate student, Eric Boder, described a yeast display system for screening combinatorial libraries ${ }^{1}$. Although not the first protein display technology to be developed-phage display and Escherichia coli display had previously been described-this was the first eukaryotic cell-based system, and as such, circumvented certain biases inherent in the prokaryotic protein machinery. In the paper, they describe the construction of a library of fusion proteins of single-chain variable fragments of antibodies to the C-terminus of yeast $\alpha$-agglutinin, the enrichment of yeast displaying the protein by flow cytometry and the selection from a mutagenized library of variants with slower 'off' times.

And Wittrup says, the method has held up to this day. His laboratory has made some minor improvements over the years, but basically, he says that you can take that 1997 paper and pretty much just follow the methods and you'll have it. In the intervening ten years, though, the technology was spun-out into a company, which stayed virtual until it was bought by BASF (now Abbott; Abbott Park, IL, USA) for \$7 million, holder of an exclusive license to the technology, and was packaged into a kit by Invitrogen (Carlsbad, CA, USA), which only just last year discontinued selling it. The kit helped get the technology into people's hands, according to Wittrup, but wasn't a big money maker for the company. All this commercial activity has not deterred researchers from using the technology — in fact the kit was meant to do just the opposite. Consequently, numerous projects by Wittrup and others have led to some successful engineered proteins and a constellation of patents emanating from the original patent.

And that's just the way Wittrup wants it. Although he saw the commercial potential and did his bit to foster that, he feels an ethical duty to disseminate the information as broadly as possible, rather than holding it close for his own financial benefit. What's more, in his view, holding it close doesn't make business sense anyway. "The best thing to do is to get as many people as possible using the technique and seeing how good it is - so that they say this is so good, we have to have it, we have to license it," he says.

This attitude may be why you see more on yeast display than some of the many other protein engineering methods, like mRNA display or E. coli display that emerged both before and after yeast display. But Wittrup thinks they are all good, although he has never been motivated to try anything else; yeast display has allowed his group to do everything they wanted to do. Still he comments, "Nobody has a monopoly on making a protein with certain properties." He does admit to a certain partisanship for his platform, however, adding that yeast display may be the best in terms of speed and probability of success. And he points to several converts to yeast display from phage and other systems-Dave Kranz of the University of Illinois, Wittrup's colleague from his days at Illinois, which holds the patents to

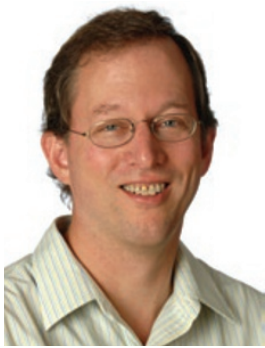

Dane Wittrup's 1997 method of yeast display holds up to this day. the technology, and the University of California at San Francisco's Jim Marks.

And where has yeast display taken Wittrup? His group has tackled several interesting human proteins - huntingtin ${ }^{2}$, interleukin 2 (ref. 3) essentially to answer the philosophical question, "Are you just a serial tool builder, or are the tools good enough to use them yourself?" But after making one particular super-binder against a tumor antigen, he found that optimized or not, the antibody wasn't persisting in the tumor, which has led him to ponder questions of pharmacokinetics and biodistribution. "It was a real wake-up call," he says. "We've moved upstream in the discovery process to what's the best way to use this thing."

1. Boder, E.T. \& Wittrup K.D. Yeast surface display for screening combinatorial polypeptide libraries. Nat. Biotechnol. 15, 553-557 (1997). (252 citations)

2. Colby, D.W. et al. Potent inhibition of huntingtin aggregation and cytotoxicity by a disulfide bond-free singledomain intracellular antibody. Proc. Natl. Acad. Sci. USA 101,17616-17621 (2004).

3. Rao, B.M. et al. High-affinity CD25-binding IL-2 mutants potentially stimulate T-cell growth. Biochemistry 44 10696-10701 (2005) 


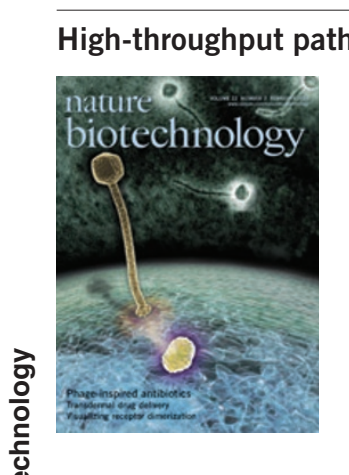

to acquisition

Drug delivery technologies generally leave a lot to be desired. Oral dosage doesn't work for large proteins, and injection is unpleasant for the patient and requires special attention from a physician. Skin-based delivery is a highly sought-after alternative. However, skin is a challenging barrier. Only a few transdermal drug delivery systems are available on the market, and they deliver only low-molecular-weight lipophilic drugs. Larger molecules such as proteins are a special challenge. The primary obstacle is low permeability through the lipid-rich matrix filled with nonviable skin cells that forms the outer layer of skin (the stratum corneum).

A large number of chemical penetration enhancers (CPEs) are known to disrupt the 윽 structure of this layer, including surfactants, fatty acids and fatty esters, but few actually improve drug transport. Moreover, those that do improve drug transport often must be used at high concentrations, increasing the odds that they will become an irritant. As a result, attempts to optimize individual enhancers usually require some compromise in safety. "You have to strike a balance," says Samir Mitragotri, professor of chemical engineering at the University of California at Santa Barbara.

Some attempts have been made to screen combinations of CPEs for synergistic effects that might improve transport at concentrations low enough to avoid irritation. The existence of hundreds of known CPEs calls for high-throughput screening techniques, as traditional screening methods are laborintensive and time consuming. To get around these problems, Mitragotri developed a new strategy based on electrical impedance as a stand-in for molecular permeability, described in his 2004 paper in Nature Biotechnology ${ }^{1}$. A decrease in electrical impedance of the skin's surface suggests that drugs will also move more freely through the barrier. Mitragotri's technique measures the electrical impedance changes caused by application of various $\mathrm{CPE}$ combinations, eliminating the time-consuming sampling steps in other techniques. "It can be measured in a few seconds, and there's no need to transfer samples. All it needs is a pair of electrodes to measure the currents," Mitragotri says.

Since the paper was published, the technology has been licensed to fqubed, now a wholly-owned subsidiary of Nuvo Research (Mississauga, ON, Canada). The system, now branded 'INSIGHT', required some tweaking. "The first thing we had to do was take this development from its academic context and apply our engineering expertise to improve the robustness and reproducibility for use in routine industrial practice. That required several iterations of development," says John M. Newsam, president and managing director of fqubed.

The high-throughput experimentation technology has allowed the company to optimize the characteristics of lead formulations, simply by allowing fqubed researchers to screen a great many more formulations than would otherwise be possible. "Samir's technology also inspired us to develop other high-throughput experimentation methods of screening the effect of materials on various properties of skin," says Newsam. "We believe if we're doing a hundred times more measurements than someone else, then the prospective product will be better."

Newsam says that the INSIGHT system was a substantial factor behind the acquisition of fqubed by Nuvo Research in 2005. Initially, fqubed undertook contract research for Nuvo. "The (fast) pace of the program" helped convince Nuvo Research to make the acquisition, he says.

Mitragotri is continuing to use the system to study the mechanism of action of CPE. "It allows us to weed out formulations that we shouldn't spend time on-[and after that] we can perform really focused studies on leading hits using conventional tools," says Mitragotri.

The work has produced some leads into mechanisms of synergy. "It's possible that the chemical enhancers interact in solution on the skin and then form a new chemical entity, or they may go in independently and work

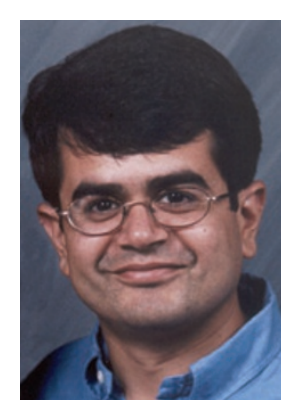

Samir Mitragotri's high-throughput methods for screening chemical enhancer combinations made a licensee a valuable target for acquisition. synergistically within the skin (barrier)," he says. In fact, experiments have provided evidence that both mechanisms may occur. "Hopefully in the near future we'll understand these mechanisms - but until then, screening is the only way to find these synergistic combinations," Mitragotri says.

1. Karande, P., Jain, A. \& Mitragotri, S. Discovery of transdermal penetration enhancers by high-throughput screening Nat. Biotechnol. 22, 192-197 (2004). (29 citations) 